\title{
APPLICATIONS OF KNOT THEORY IN FLUID MECHANICS
}

\author{
RENZO L. RICCA \\ Department of Mathematics, University College London \\ Gower Street, London WC1E 6BT, U.K. \\ E-mail: ricca@math.ucl.ac.uk
}

\begin{abstract}
In this paper we present an overview of some recent results on applications of knot theory in fluid mechanics, as part of a new discipline called 'topological fluid mechanics' (TFM). The choice of the topics covered here is deliberately restricted to those areas that involve mainly a combination of ideal fluid mechanics techniques and knot theory concepts, complemented with a brief description of some other concepts that have important applications in fluid systems. We begin with the concept of topological equivalence of fluid flow maps, giving a definition of knotted and linked flux-tubes. In the fluid mechanics context Reidemeister's moves are interpreted in terms of local actions of fluid flows performed on fluid structures. An old theorem of Lichtenstein (1925) concerning the isotopic evolution of vortex structures in the context of the Euler equations is re-proposed and discussed in the TFM context for the first time. Then, we review the relationship between helicity and linking numbers and we present some recent results on magnetic relaxation of linked, knotted and braided structures in magnetohydrodynamics. In the context of the Euler equations (and under certain approximations given by the so-called 'localized induction' for vortex structures) we briefly examine some interesting relationships between integrability and existence and stability of vortex filaments in the shape of torus knots. We conclude with an overview of some new results concerning electrically charged knots embedded in a viscous fluid, elastic relaxation of strings and braids and relationships between energy levels and topological information. Some simple bounds on elastic energy levels given by global geometric quantities and topological quantities are presented and discussed for the first time.
\end{abstract}

1. Modern developments in topological fluid mechanics. Knotted and linked structures are ubiquitous in nature and in fluid flows in particular. Their scale lengths range from $10^{-10} \div 10^{-6} \mathrm{~m}$, for tiny macromolecules, polymers, defect lines and superfluid vortices, to $10^{-2} \div 10^{2} \mathrm{~m}$, for fluid eddies, vortex filaments, tornadoes, and up to $10^{6} \div$ $10^{10} \mathrm{~m}$ for gigantic magnetic flux-tubes, plasma loops and magnetic arches present in

1991 Mathematics Subject Classification: Primary 76-02; Secondary 54M99.

Financial support from The Leverhulme Trust is kindly acknowledged.

The paper is in final form and no version of it will be published elsewhere. 
stellar atmospheres and in astrophysical flows. Observational and experimental evidence of complex braided and entagled fluid structures is now well documented in literature, in the context of classical fluid mechanics (see, for example, Lugt, 1983; Van Dyke, 1988) and magnetohydrodynamics (see, for example, Bray et al., 1991).

Since the original result of Gauss (1833) and the work of Maxwell (1841-1873) on linked electric circuits and magnetic fields, the studies of Kelvin $(1869 ; 1875)$ on knotted vortices, and the first mathematical classification of knots by Tait (1898), applications of knot theory in fluid mechanics have become an integral part of what is now called 'topological fluid mechanics' (TFM). After little more than a century, topological fluid mechanics has become a diversified and rich discipline (see the proceedings edited by Moffatt \& Tsinober, 1990; Moffatt et al., 1992; Kauffman, 1995a; 1995b; and the review article by Ricca and Berger, 1996). Fluid mechanics deals essentially with continuous deformations of fluid structures; hence, it provides a natural setting for useful applications of ideas and results coming from topology and geometry. In turn, topological fluid mechanics offers many stimulating problems to applied mathematicians and knot theorists.

1.

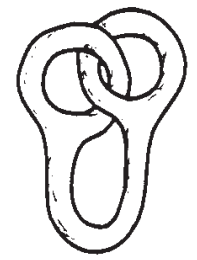

2 .

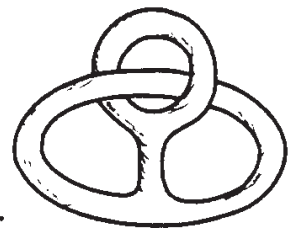

3.

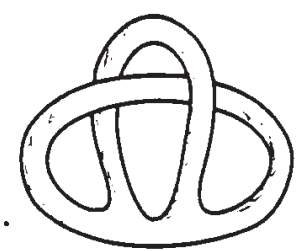

5.

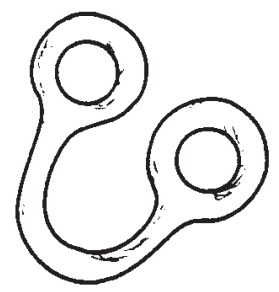

4.

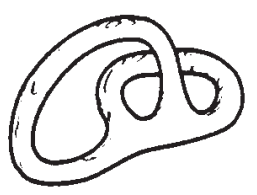

Fig. 1. In the context of the Euler equations fluid structures can be deformed without change of topology. A fluid structure may undergo a sequence of 'pretzel transformations' that leave the structure in the same topological equivalence class. The figure shows 5 possible configurations attained by a fluid structure in an ideal fluid (states $1,2, \ldots, 5$ are topologically equivalent).

There are four major active research areas.

1.1. Search for knotted solutions to Euler's equations. The motion of fluid structures (e.g. vortex filaments) embedded in a homogeneous, uniform and perfectly inviscid fluid is governed by the Euler equations

$$
\frac{\partial \mathbf{u}}{\partial t}+(\mathbf{u} \cdot \nabla) \mathbf{u}=-\frac{1}{\rho} \nabla p
$$

where $\mathbf{u}=\mathbf{u}(\mathbf{X}, t)$ denotes the velocity field, smooth function of the position vector $\mathbf{X}$ and time $t, \rho$ the fluid density and $p$ the fluid pressure. Euler's equations are a special case of the Navier-Stokes equations at zero viscosity. In the inviscid context the topology of fluid flow and structures is conserved (see figure 1); knot theory is applied to study and 
classify fluid patterns given by evolution of streamlines (integral curves that at each time have same direction as the velocity vector $\mathbf{u}$ at each point $\mathbf{X}$ ) and vorticity lines (integral curves that at each time have same direction as the vorticity vector $\omega=\nabla \times \mathbf{u}$ at each point $\mathbf{X}$ ). There are many open questions about integrability, existence of singularities and existence and stability of global solutions in terms of vortex structures (for example, the existence and stability of knotted vortex tubes). To tackle these problems a topological approach has shown to be very helpful and innovative (see Moffatt et al., 1992). Moreover, the braiding and entanglement of vortex structures are examples of physical mechanisms potentially important for the re-distribution of energy and helicity in the bulk of the fluid (see, for example, the proceedings edited by Jiménez, 1991), and in the last years there has been a growing interest to study these structures using topological techniques.

Under Euler's equations a number of physical quantities such as kinetic energy, linear and angular momentum, helicity, etc., are conserved during evolution. It is very important to understand the relationship between these physical conserved quantities and known topological invariants. This approach, initiated by Moffatt (1969) with his seminal work on the topological interpretation of helicity in terms of (Gauss) linking number, is a very interesting and promising area of research (Khesin \& Chekanov, 1989; Tur \& Yanovsky, 1993).

1.2. Relationships between energy relaxation and knot topology. Knotted and linked fluid structures deform continuously to a state that is energetically favourable. During this relaxation process we may assume (and there are cases in which this is a physical requirement) that the knot topology remains conserved, so that the physical knot (or link) can relax to some minimum energy configuration, while remaining in the same class of topological equivalence. In ideal magnetohydrodynamics (MHD), for example, the topology of magnetic field structures is 'frozen' during evolution. Physical conserved quantities and topological invariants such as linkage, knot type, braiding, etc., form therefore a large family of quantities that remain conserved in the fluid medium. In the relaxation of magnetic structures, driven by the magnetic tension induced by the Lorentz force, these invariants 'guide' the magnetic field towards a state of minimum energy by acting as constraints of the relaxation process (Moffatt, 1992). Since the topology of a magnetic configuration is frozen in ideal conditions, initial states and end states (i.e. minimum energy states) are confined to the same class of topological equivalence. Clearly, there is no guarantee that initial states with different geometry and same topology will eventually evolve to the same configuration (uniqueness of the solution): it has been suggested (Moffatt, 1990) that the relaxation will lead to a spectrum of equilibrium configurations that are topologically equivalent, but different in geometry and energy levels. Magnetic braids, knotted elastic strings and electrically charged knots, are all examples of physical knots that are studied by minimising appropriate energy functionals. The results of these studies find also useful applications in applied science and engineering.

1.3. Studies of dynamical systems and measure preserving flows. Topological properties of three-dimensional vector fields play an important part in the study of trajectories of autonomous differential equations in $\mathbb{R}^{3}$ (Birman \& Williams, 1983; Holmes \& Williams, 1985; Bedford \& Swift, 1988). In this context, a typical question is to ask whether for a 
given vector field with some closed orbits forming a link, one can deduce existence of other closed orbits linked with the former; the existence of chaotic orbits is also an important problem. Existence theorems for knotted orbits of flows in a solid torus have been derived, as well as lower bounds for the topological entropy of such orbits. Many of these results are based on the Nielsen-Thurston theory of isotopy classes of surface homeomorphisms (Meyer \& Saari, 1988) and are valid for vector fields that are divergence-free. Interesting applications of knot theory to flow systems exhibiting pitchfork, period doubling and bifurcations, as well as applications of asymptotic linking concepts to space filling curves, form now an integral part of current research topics relevant to TFM (see, for example, Uezu, 1990; Arnold \& Khesin, 1992). Other open questions regard transport properties by three-dimensional volume-preserving vector fields and relationship between topology of ideal fluid flows and their Hamiltonian structure (Kuznetsov \& Mikhailov, 1980; Marsden \& Weinstein, 1983).
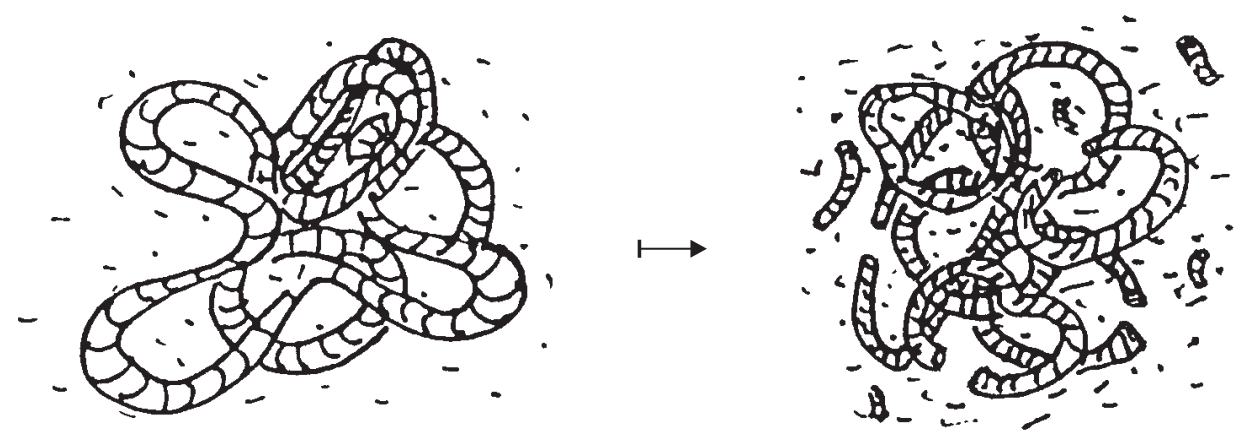

Fig. 2. Fluid structures may undergo a natural change in topology when dissipative effects (due to fluid viscosity or plasma resistivity) are present. Topological change is due to local processes of physical reconnection of different parts of fluid structures. In a first, simple approximation these events may be modelled by techniques of 'oriented surgery' on the constitutive vector fields.

1.4. Physical reconnection mechanisms and oriented surgery. When dissipative effects are present (due to fluid viscosity or plasma resistivity) fluid structures undergo a topological change due to physical reconnections (see figure 2). Simple mathematical modelling of these processes involves techniques of 'oriented surgery' performed on the bundle of the constitutive vector fields, integrated by some knowledge of the particular physical process (see, for example, the review by Kadomtsev, 1987 on reconnection of magnetic fields, and the paper by Boratav et al., 1992 on reconnection of vortex filaments). At the moment there is no accurate description of the physical mechanism of reconnection, and many studies are the result of direct numerical simulations of interacting fluid structures (interaction of vortex filaments or magnetic flux-tubes) in the context of the Navier-Stokes equations or in the context of the resistive magnetohydrodynamical equations. For example, numerical work carried out by Aref and Zawadzki (1991) reveals the linking of two colliding vortex rings. These numerical experiments give also important information on the robustness of fluid and topological invariants under the action 
of dissipative effects, a key issue to understand the energy dissipation in real physical systems.

In this context, knot theory is very useful for diagnostic purposes, and there are cases (for instance in the magnetic field context) in which estimates of energy release through reconnection are based on crossing number techniques, topological information and combinatorics (see, for example, Freedman \& Berger, 1993). Formation of singularities in the flow field, appearance of bifurcations in the corresponding orbits, and development of chaos are of course fundamental related aspects, in which knot theory plays also an increasingly important role.

In the following sections we shall discuss and review some results concerning the first two areas of research. We believe that these results provide a good example of new applications of knot theory in fluid mechanics, and are part of modern developments in topological fluid mechanics.

2. Topological equivalence classes for frozen fields. Let us consider a homogeneous, inviscid (i.e. ideal), incompressible and perfectly conducting fluid in a domain $\mathcal{D} \subseteq \mathbb{R}^{3}$. Fluid motion is described by the velocity vector field $\mathbf{u}=\mathbf{u}(\mathbf{X}, t)$, smooth function of the vector position $\mathbf{X}$ and time $t$, satisfying

$$
\nabla \cdot \mathbf{u}=0 \quad \text { in } \mathcal{D}, \quad \mathbf{u}=0 \quad \text { on } \partial(\mathcal{D})_{\infty},
$$

where $\partial(\mathcal{D})_{\infty}$ denotes the boundary of $\mathcal{D}$ (possibly at infinity).

A Lagrangian description of fluid motion is given by the functional relation $\mathbf{X}=$ $\mathbf{X}(\mathbf{a}, t)$, where $\mathbf{a}$ is the initial position of a fluid particle. The velocity field induces a fluid flow map $\varphi=\varphi(\mathbf{X}, t)$ that maps each fluid particle instantaneously, from the initial position $\mathbf{a}$ to the final position $\mathbf{X}$ by

$$
\varphi_{t}: \mathbf{a} \longmapsto \mathbf{X}, \quad t \in I .
$$

We assume the flow map to be smooth, have an inverse and be volume-preserving, i.e.

Definition 1. The fluid flow map is a functional element

$$
\varphi \in\left\{C^{\infty}: \exists \varphi^{-1} \mid \mathbf{a}=\varphi^{-1}(\mathbf{X}, t) \quad \forall t \in I ; \varphi \text { volume-preserving }\right\} .
$$

This means that under the action of the flow map $\varphi$ a sub-domain $\mathcal{V}$ of fluid volume, bounded by a smooth surface $\mathcal{S}=\partial \mathcal{V}$, moves in the fluid changing shape, while keeping volume constant. Here we consider only 'tame' flow maps, i.e. flow maps that are 'nondegenerate'; flow maps that involve multivaluedness or discontinuity in the mapping are considered 'degenerate' and are not discussed in this paper. We should point out, though, that flow maps associated with degenerate flow patterns, with formation of cusps, vector field bifurcations, singularities, etc., are ubiquitous and very important for mathematical modelling of physical reconnections and description of changes in topology.

Let us consider a magnetic field $\mathbf{B}=\mathbf{B}(\mathbf{X}, t)$ embedded in the fluid domain. Let $\mathbf{B}$ be solenoidal (i.e. $\nabla \cdot \mathbf{B}=0$ ) in $\mathcal{D}$. Consider a bundle of magnetic field lines, each line being the integral curve that at each time has same direction as the magnetic field $\mathbf{B}$ at $\mathbf{X}$. We have 
Definition 2. The magnetic flux $\Phi$ is the surface integral defined by

$$
\Phi=\int_{\mathcal{S}} \mathbf{B} \cdot \mathbf{n} d A
$$

where $\mathcal{S}$ is a simply connected two dimensional surface of area $A$, bounded by a simple, unknotted, closed circuit $\mathcal{C} \equiv \partial \mathcal{S}$ (encircling $\mathbf{B}$ once), and $\mathbf{n}$ is the normal to $\mathcal{S}$ pointing in the positive direction induced by the $\mathbf{B}$-field.

Suppose the magnetic field is convected with the fluid flow under the condition that the flux of $\mathbf{B}$ through any closed circuit moving with the fluid is conserved. Since there is no dissipation and no diffusion of magnetic field lines, then the magnetic field is 'frozen' in $\mathcal{D}$ (up to continuous deformations) and transported in the fluid medium by the velocity field $\mathbf{u}$, i.e.

Definition 3. A vector field $\mathbf{B}$ is said to be frozen in $\mathcal{D}$ if and only if it satisfies the frozen field equation

$$
\frac{\partial \mathbf{B}}{\partial t}=\nabla \times(\mathbf{u} \times \mathbf{B}) .
$$

When we replace the $\mathbf{B}$-field with the vorticity field $\omega=\nabla \times \mathbf{u}($ with $\nabla \cdot \omega=0$ in $\mathcal{D}$ ), eq. (6) becomes the vorticity transport equation in the Euler equations context. In this case we have the crucial non-linear coupling between vorticity and velocity field, through the curl operator. A formal property of equation (6) (valid in kinetic and magnetic contexts) is represented by the integral solutions given by the Cauchy equations

$$
B_{i}(\mathbf{X}, t)=B_{j}(\mathbf{a}, 0) \frac{\partial X_{i}}{\partial \mathrm{a}_{j}}
$$

which encapsulate both the convection of the field from the initial position $\mathbf{a}$ to $\mathbf{X}$, and the simultaneous rotation and distortion of fluid elements by the deformation tensor $\partial X_{i} / \partial \mathrm{a}_{j}$. Since this tensor is a time-dependent diffeomorphism of position, it maps continuously the initial field distribution from $\mathbf{B}(\mathbf{a}, 0)$ to $\mathbf{B}(\mathbf{X}, t)$, establishing a topological equivalence between the two fields; hence, we write

$$
\mathbf{B}(\mathbf{a}, 0) \sim \mathbf{B}(\mathbf{X}, t) .
$$

Note that since the fluid is assumed to be incompressible, the Jacobian of the transformation has $\operatorname{det}\left(\partial X_{i} / \partial \mathrm{a}_{j}\right)=1$.

3. Knotted and linked flux-tubes. A magnetic flux-tube embedded in $\mathcal{D}$ corresponds to the intuitive idea of a coherent bundle of closed magnetic field lines to form a tube-like region. If the tube axis (which is a magnetic line) is in the shape of a knot, the magnetic flux-tube, formed by the magnetic tubular neighbourhood of field lines, is also knotted. Formally, knotted and linked magnetic flux-tubes are constructed in a standard way as indicated, for example, by Moffatt (1990) and Moffatt \& Ricca (1992; see also the reprint in Kauffman, 1995a). The mathematical procedure is based on the idea of embedding a bundle of magnetic lines to constitute a tubular neighbourhood of the unknot and then, by a combination of Dehn's surgery, isotopy and appropriate cross-switchings, obtain the desired linked (knotted) magnetic flux-tube. The resulting physical knot can be defined in a simple way. Let us consider first the following 
Definition 4. A surface $\mathcal{S}$ is a magnetic surface in $\mathcal{D}$ if it is made of magnetic lines that are tangent everywhere to the surface in $\mathcal{D}$.

Since $\mathbf{B}$ is frozen during evolution, it follows that if $\mathcal{S}$ is a magnetic surface at time $t=0, \varphi_{t}(\mathcal{S})$ is a magnetic surface for all time $t \geq 0$.

Consider now the standard solid torus $T$ in $\mathbb{R}^{3}$ given by

$$
((2+\epsilon \cos \beta) \cos \alpha,(2+\epsilon \cos \beta) \sin \alpha, \epsilon \sin \beta),
$$

where $\alpha \in[0,2 \pi), \beta \in[0,2 \pi)$ and $\epsilon \in[0,1]$. Let $\mathcal{F}_{p, q}(p>q>1$ co-prime integers $)$ denote the foliation of $T$ by the curves $\Gamma_{\epsilon, \beta}$ given by

(10) $\Gamma_{\epsilon, \beta}(s)=[(2+\epsilon \cos (\beta+q s)) \cos (p s),(2+\epsilon \cos (\beta+q s)) \sin (p s), \epsilon \sin (\beta+q s)]$, where $s \in[0,2 \pi)$. Lomonaco (1995) suggests the following

Definition 5. A collection of linked (knotted) magnetic flux-tubes $\mathcal{L}_{m}$ is defined as a smooth embedding into $\mathcal{D}$ of finitely many disjoint standard solid tori $\sqcup_{i} T_{i}$ and a smooth magnetic field $\mathbf{B}$ such that:

(i) $\mathcal{L}_{m}$ is an embedding when restricted to the interior of $\sqcup_{i} T_{i}$;

(ii) the bounding surface $\cup_{i} \mathcal{L}_{m}\left(\partial T_{i}\right)$ is a magnetic surface;

(iii) for each component $\mathcal{L}_{m}\left(T_{i}\right)$ there exists a pair $\left\{p_{i}, q_{i}\right\} \quad\left(p_{i}>q_{i}>1\right.$ co-prime integers) such that $\mathcal{L}_{m}$ maps the foliation $\mathcal{F}_{p_{i}, q_{i}}$ of $T_{i}$ onto the integral curves of $\mathbf{B}$ in $\mathcal{L}_{m}\left(T_{i}\right)$.

If $p$ and $q$ are real numbers, $p / q$ is irrational and we can extend the definition to include linked magnetic flux-tubes formed by non-closed integral curves of $\mathbf{B}$ given by $\Gamma_{\epsilon, \beta}$ space-filling the tori $\sqcup_{i} T_{i}$ (Arnold, 1974).

Since the magnetic field is frozen in $\mathcal{D}$, linked (knotted) magnetic flux-tubes (magnetic links and knots) are also frozen and convected in the fluid by isotopies, within the topological equivalence class of the link (knot) type. Moreover, since the action of the flow map is volume-preserving, the magnetic volume of each flux-tube, given by

$$
V_{i}=V_{i}\left[\varphi_{t}\left(\mathcal{L}_{m}\left(T_{i}\right)\right)\right]=\int_{\varphi_{t}\left(\mathcal{V}_{i}\right)} d V_{i}
$$

where $\varphi_{t}\left(\mathcal{V}_{i}\right)$ is the magnetic volume of $T_{i}$ under $\varphi$, is constant at any time $t \in I$. This means that the associated fluid mass $m_{i}=m_{i}\left(V_{i}\right)$ is also conserved (because the fluid density $\rho$ is constant). Volume and mass are therefore two scalar, physical invariants associated with the fluid evolution of physical knots. Another important invariant is the magnetic flux. For each $T_{i}$ let us choose a meridional disk $\mathcal{S}_{i}$ of area $A_{i}$ (in the cross-sectional plane).

Definition 6. The magnetic flux $\Phi_{i}=\Phi_{i}\left(\mathcal{L}_{m}\left(T_{i}\right)\right)$ associated with the $i$-th link component is the surface integral defined by

$$
\Phi_{i}=\Phi_{i}\left(\mathcal{L}_{m}\left(T_{i}\right)\right)=\int_{\mathcal{L}_{m}\left(\mathcal{S}_{i}\right)} \mathbf{B} \cdot \mathbf{n} d A_{i}
$$

where $\mathbf{n}$ is the normal to $\mathcal{L}_{m}\left(\mathcal{S}_{i}\right)$ pointing in the positive direction induced by the $\mathbf{B}$-field.

Moreover, the following result holds true: 
THEOREM 1 (Helmholtz, 1858; Alfvén, 1950). Under frozen field evolution the magnetic flux $\Phi_{i}$ associated with the $i$-th link component is conserved, i.e.

$$
\frac{d}{d t} \Phi_{i}\left(\varphi_{t}\left(\mathcal{L}_{m}\right)\right)=0
$$

In vortex dynamics this result is known as Helmholtz's III law of vortex motion (replacing $\mathbf{B}$ with $\omega$ ), and represents a fundamental result in the mathematical theory of ideal fluid mechanics. Alfvén proved this result in ideal magnetohydrodynamics, almost hundred years later.

4. Reidemeister's moves and Lichtenstein's theorem. Topological fluid mechanics deals essentially with the study of fluid structures (thought of as embeddings) that can be continuously deformed one into another by ambient isotopies. Since $\varphi$ is both continuous and has an inverse, that is also continuous with respect to $t$, then $\varphi_{t_{1}}\left(\mathcal{L}_{m}\right)$ and $\varphi_{t_{2}}\left(\mathcal{L}_{m}\right)$ are ambient isotopic for $\left\{t_{1}, t_{2}\right\} \in I$ in $\mathcal{D}$, and we write $\mathcal{L}_{m, t_{1}} \sim \mathcal{L}_{m, t_{2}}$. Ambient isotopy generates equivalence classes of linked and knotted magnetic flux-tubes. Continuous deformations induced by fluid actions convect flux-tubes in $\mathcal{D}$. One of the simplest, natural deformations is given by the stretching of flux-tubes. If natural stretching takes place, the total length of the tube axis increases, and by conservation of magnetic volume and flux, we have a corresponding decrease of the tube cross-section and an increase of magnetic field intensity. From a geometric viewpoint, this simple deformation corresponds to a trivial time-dependent, continuous, re-parametrization of the tube centreline, with a continuous deformation of the integral curves in $\mathcal{L}_{m}\left(T_{i}\right)$. This re-parametrization (via homotopy maps) provides a simple example of ambient isotopy for the magnetic link (knot) $\mathcal{L}_{m}$.

Knot topology is preserved under the action of the Reidemeister moves (see, for example, Kauffman, 1987). In the context of the Euler equations these moves are performed quite naturally by the action of local flows on the strands of fluid structures (see figure 3 ). If the background fluid in the complement $\left(\mathcal{D}-\mathcal{L}_{m}\right)$ is irrotational (and at rest), then these flows must satisfy the Dirichlet problem for the Laplacian of the stream function $\psi$, given by

$$
\left\{\begin{array}{l}
\mathbf{u}=\nabla \psi \\
\nabla^{2} \psi=0
\end{array} \quad \text { in } \quad\left(\mathcal{D}-\mathcal{L}_{m}\right)\right.
$$

with boundary conditions

$$
\begin{cases}\mathbf{u} \cdot \mathbf{n}=\mathbf{u}_{\perp} & \text { on } \cup_{i} \mathcal{L}_{m}\left(\partial T_{i}\right), \\ \mathbf{u}=0 & \text { on } \partial(\mathcal{D})_{\infty},\end{cases}
$$

where $\mathbf{u}_{\perp}=\mathbf{u}_{\perp}(\mathbf{X}, t)$ is the normal component of the velocity on the bounding magnetic surface, with $\mathbf{n}$ denoting the normal vector to the surface. Equations (14) and (15) have a unique solution in terms of local flows (Batchelor, 1967). These flows correspond to Reidemeister's moves through a sequence of continuous deformations of the tube strands. Note that in the boundary condition (15) there is no prescription for the tangential component of the velocity on the bounding magnetic surface. As we pointed out earlier, tangential effects (present, for instance, if the flux-tube gets stretched) induce merely 

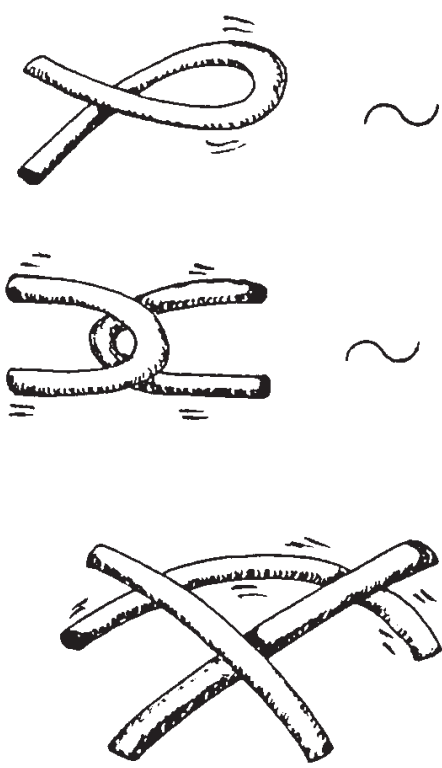
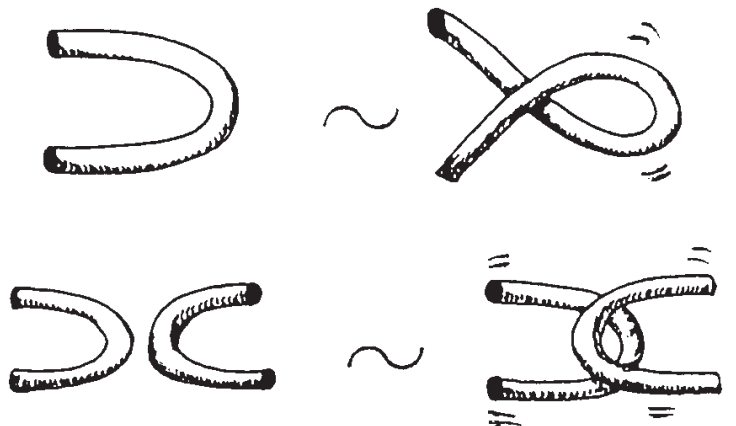

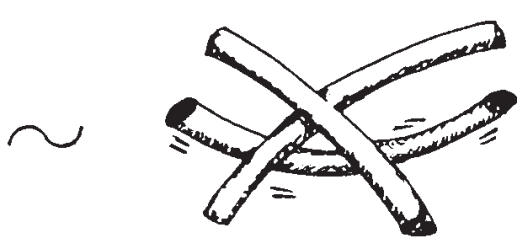

Fig. 3. The three types of Reidemester's moves (see, for example, Kauffman, 1987) are performed naturally by the action of local fluid flows on the tube strands of magnetic and vortex structures.

ambient isotopies of fluid structures, hence preserving the topology of the magnetic link (knot). This means that Reidemeister's moves are performed by local flows that are solutions to (14-15), up to arbitrary tangential actions.

For linked (knotted) vortex filaments $\mathcal{L}_{v}$ (the analogues of magnetic flux-tubes, when vorticity replaces the magnetic field), the conditions for having ambient isotopies are given by a theorem due to Lichtenstein, formulated in 1925 (see also Villat, 1930).

For simplicity let us consider just one, isolated vortex filament (a vortex knot) $\mathcal{L}_{v}(T) \equiv$ $\mathcal{K}_{v}(T)$ in $\mathcal{D}$. Let $\mathbf{v}=\left(v_{1}, v_{2}, v_{3}\right)$ denote the velocity of $\mathcal{K}_{v}$, with boundary $\mathcal{K}_{v}(\partial T)$ (a vortex surface), and with $\mathbf{v}$ and $\mathcal{K}_{v}(\partial T)$ sufficiently smooth (at least of class $C^{2}$ ), and $v_{i} \propto|\mathbf{X}|^{-2}$ as $|\mathbf{X}| \rightarrow \partial(\mathcal{D})_{\infty}$. We have:

Theorem 2 (Lichtenstein, 1925). Let $\mathcal{K}_{v}(T)$ be a vortex filament in $\mathcal{D}$ of velocity $\mathbf{v}$ and bounding surface $\mathcal{K}_{v}(\partial T)$, satisfying the conditions $K_{a}\left(v_{i}\right)$ and $K_{a}\left(\mathcal{K}_{v}(\partial T)\right)$, where

$$
K_{a}(\cdot) \equiv\left|\partial_{i}^{2}(\cdot)_{1}-\partial_{i}^{2}(\cdot)_{2}\right| \leq b\left|\mathbf{X}_{1}-\mathbf{X}_{2}\right|^{a} \quad i=1,2,3,
$$

with $b>0$ and $0<a<1$ constants, and $\left\{\mathbf{X}_{1}, \mathbf{X}_{2}\right\} \in \mathcal{K}_{v}(\partial T)$. Then, at any finite time $t \in I$ we have:

(i) $\mathcal{K}_{v, t_{1}}$ is topologically equivalent to $\mathcal{K}_{v, t_{2}}$ in $\mathcal{D}$, for any $\left\{t_{1}, t_{2}\right\} \in I$;

(ii) $\mathbf{v}$ is determined uniquely in $\mathcal{D}$;

(iii) $K_{a}\left(v_{i}\right)$ holds true at any time $t \in I$.

The proof of this theorem can be found in the original paper by Lichtenstein and is also given in the XII chapter of Villat's book (1930, pp. 256-269). The proof is based 
on the Cauchy equations for a divergence-free velocity field. In the light of a topological approach to fluid mechanics, Lichtenstein's result strikes for its modern interpretation of convection of vortex structures under Euler flows. In this sense Lichtenstein's theorem may well be considered one of the first examples of topological interpretation of fluid mechanical properties.

5. Helicity and linking numbers. In ideal fluids many physical and topological quantities are invariant of the evolution. Magnetic volume, fluid mass and magnetic flux are the three physical invariants discussed in the previous section. Other physical invariants are total energy, linear and angular momentum and helicity. Example of topological invariants are knot and link type, braiding group and linking numbers. A fundamental question in topological fluid mechanics is to establish whether physical and topological invariants are related one another and how. Let us consider helicity.

Definition 7. The helicity of a linked (knotted) magnetic flux-tube $\mathcal{L}_{m}$ in $\mathcal{D}$ is defined by

$$
H\left(\mathcal{L}_{m}\right)=\int_{\cup_{i} \mathcal{L}_{m}\left(T_{i}\right)} \mathbf{A} \cdot \mathbf{B} d V_{i},
$$

where $\mathbf{B}=\nabla \times \mathbf{A}$ is the magnetic field, $\mathbf{A}$ is the vector potential (with $\nabla \cdot \mathbf{A}=0$ ).

Helicity is just a fluid dynamical expression of the Hopf integral, i.e. the integral of the scalar product of a solenoidal vector field and its curl; the first proof that this quantity is an isotopy invariant was given by Whitehead (1947). However, in the context of fluid mechanics conservation of helicity was shown much later, with the works of Woltjer (1958) and Moreau (1961), and the result was further extended by Arnold (1974) in his discussion of asymptotically space-filling curves. We have:

Theorem 3 (Woltjer, 1958; Moreau, 1961). The helicity of $\mathcal{L}_{m}$ is an invariant of the Euler equations, that is

$$
\frac{d}{d t} H\left(\varphi_{t}\left(\mathcal{L}_{m}\right)\right)=0, \quad \text { in } \mathcal{D}
$$

This result has been extended to give a topological interpretation of helicity in terms of linking numbers. In summary, we have:

Theorem 4 (Moffatt, 1969; Berger \& Field 1984, Moffatt \& Ricca 1992). Let $\mathcal{L}_{m}$ be a collection of magnetic links (knots). Then

$$
H\left(\mathcal{L}_{m}\right)=\sum_{i} L k_{i} \Phi_{i}^{2}+2 \sum_{i \neq j} L k_{i j} \Phi_{i} \Phi_{j},
$$

where $L k_{i}$ denotes the (Călugăreanu-White) linking number of the tube axis in $\mathcal{L}_{m}\left(T_{i}\right)$ with respect to the framing induced by the embedding of the $\mathbf{B}$-field, and $L k_{i j}$ denotes the (Gauss) linking number of $\mathcal{L}_{m}\left(T_{i}\right)$ with $\mathcal{L}_{m}\left(T_{j}\right)$.

This result establishes a fundamental relationship between physical conserved quantities (helicity and flux) and topological invariants (linking numbers). Let $\mathcal{L}_{m}(T) \equiv \mathcal{K}_{m}(T)$ 
denote a one-component magnetic link (a knotted magnetic flux-tube). Then, equation (19) reduces to

$$
H\left(\mathcal{K}_{m}\right)=L k \Phi^{2}=(W r+T w) \Phi^{2},
$$

where the (Călugăreanu-White) linking number $L k$ is decomposed into $W r$, the writhing number of the tube axis, and $T w$, the total twist of the flux-tube (for a precise definition of these quantities see Călugăreanu, 1961; White, 1969; see also Moffatt \& Ricca, 1992). Equation $(20)$ shows that $H\left(\mathcal{K}_{m}\right)$ can be decomposed into writhe and twist contributions. Although the sum of these two quantities is a topological invariant, the writhe and twist contributions, taken separately, are not invariant (they are purely geometric quantities) and their values change with a change of shape. This means that $W r$ and $T w$ interchange continuously under isotopy.

The writhing number $W r$ is characterised by the following properties:

i) $W r$ depends only on the geometry of the tube axis;

ii) $W r$ is invariant under rigid motions or dilations of $\mathcal{D}$ (conformal invariant), but its sign is changed by reflection;

iii) in passing from an under-crossing to an over-crossing of the strands (in some projection plane), its value jumps by +2 .

It can be shown (Fuller, 1971; Moffatt \& Ricca, 1992) that the writhe admits physical interpretation in terms of the sum of signed crossings of the diagram of the tube axis in some projection plane, averaged over all projections, that is

$$
W r=<n_{+}(\nu)-n_{-}(\nu)>,
$$

where the angular brackets denote averaging over all directions $\nu$ of projection, and $n_{ \pm}$ denotes the number of apparent \pm crossings, from the direction of projection $\nu$. For a nearly plane curve (except small indentations to allow crossings) the writhe can be directly estimated by the sum of the signed crossings.

The total twist $T w$ is given by the sum of the total torsion of the tube axis and the intrinsic twist of the B-lines in the tube $T$, divided by $2 \pi$ (Moffatt \& Ricca, 1992), and has the following properties:

i) $T w$ is a continuous function of the tube-axis;

ii) $T w$ is invariant under rigid motions or dilations of $\mathcal{D}$ (conformal invariant), but its sign is changed by reflection;

iii) $T w$ is additive for contiguous tubular segments of $T$.

Part of the twist contribution to helicity is associated with the torsion of the tube axis and part with what may be described as 'intrinsic twist' of the field lines in the flux-tube. This decomposition and the relation between Reidemeister's type I move and kinetic helicity (defined similarly by the integral of $\mathbf{u} \cdot \omega$ over the vortex volume) of a vortex filament $\left(\mathcal{L}_{v} \equiv \mathcal{K}_{v}\right)$ are discussed in Ricca \& Moffatt (1992). 
On the other hand, if the embedding of the B-lines corresponds to a zero-framing of each component $\mathcal{L}_{m}\left(T_{i}\right)$ (i.e. $L k_{i}=0$ for each component), then

$$
H\left(\mathcal{L}_{m}\right)=2 \sum_{i, j} L k_{i j} \Phi_{i} \Phi_{j}
$$

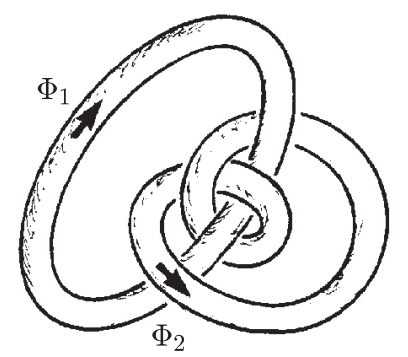

Fig. 4. Example of two (zero-framed) linked flux-tubes with zero (Gauss) linking number. The helicity of the system is zero!

As is shown in figure 4, there are cases of non-trivial links with zero (Gauss) linking number and zero helicity. It is interesting to note that Maxwell (1873, vol. II, p. 43) was aware of this fact, noticing that the so-called 'Whitehead' link had indeed $L k_{12}=0$, as he remarked in his study of linked electric circuits.

Higher-order linking numbers able to capture complex topologies (as in the case of the Borromean rings) have been introduced by Berger (1990; 1991; see also Evans \& Berger, 1992). Since links are close relatives of braids, a hierarchy of linking integrals, the first member of which is just the Gauss integral $L_{i j}$, generates a family of winding numbers for braids. These last invariants admit physical interepretation in terms of helicity integrals for braided magnetic flux-tubes expressed through higher-order braid linkings.

6. Magnetic relaxation under topological constraints. Consider now a perfectly conducting, incompressible and viscous fluid in $\mathcal{D}_{1}$, where magnetic links (knots) $\mathcal{L}_{m}$ are present. Magnetic flux-tubes are subject to the Lorentz force $\mathbf{F}$, induced by the magnetic field, that in a first approximation is given by $\mathbf{F} \propto c \mathbf{n}$, where $c$ is the curvature of the tube axis and $\mathbf{n}$ the principal unit normal. This force induces a shrinking of the tube length and is equivalent to the action of a 'shortening flow' on $\mathcal{L}_{m}$. Curvature effects are present through a longitudinal tension in the tube that makes it behaving like a rubber band in tension (with magnetic waves propagating along the tube as elastic waves on strings).

Definition 8. The magnetic energy of $\mathcal{L}_{m}$ is defined by

$$
E_{m}(t)=E_{m}\left(\varphi_{t}\left(\mathcal{L}_{m}\right)\right)=\frac{1}{2} \int_{\cup_{i} \mathcal{L}_{m}\left(T_{i}\right)}|\mathbf{B}|^{2} d V_{i} .
$$

In this case the fluid is dissipative and magnetic energy is not conserved during evolution; instead, it is converted into kinetic energy and then dissipated by internal friction. 
This relaxation process for magnetic energy is governed by the Navier-Stokes equations. Often magnetic relaxation is analysed by means of model equations that are simpler than the Navier-Stokes equations, while being representative of the correct dynamics (see, for example, Moffatt, 1992).

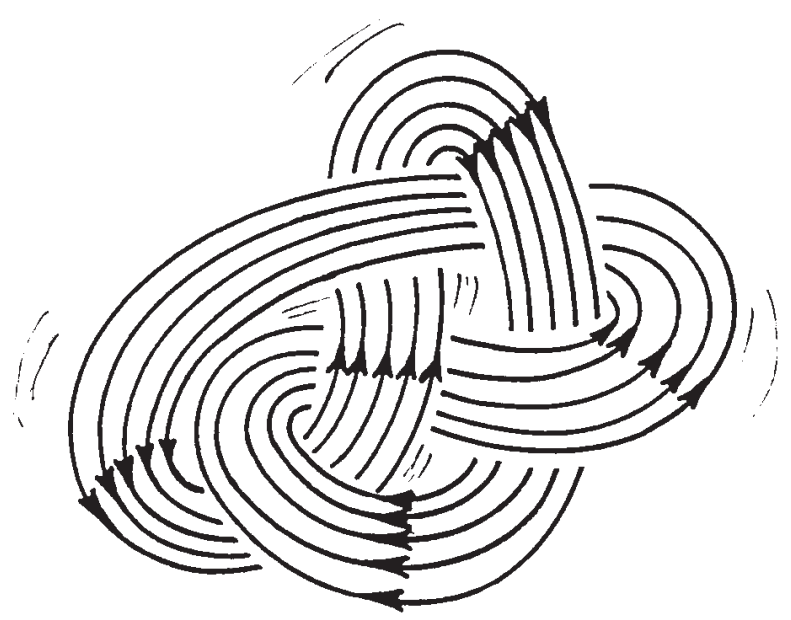

Fig. 5. Relaxation of knotted magnetic field lines. The process is governed by a 'shortening flow' acting on the system of field lines guided by the relaxation of magnetic tension. The process comes to a halt when different parts of $\mathcal{L}_{m}$ come into contact with each other.

Under these model equations it can be shown that the magnetic link (knot) passes from high to low magnetic energy levels according to

$$
\frac{d E_{m}}{d t} \propto-\int_{\cup_{i} \mathcal{L}_{m}\left(T_{i}\right)}|\mathbf{u}|^{2} d V_{i},
$$

by relaxing internal magnetic tension; during relaxation, magnetic volume and flux are still preserved (viscosity does not effect the magnetic field), while B-lines are being shortened by internal tension. Since this evolution is volume-preserving, the natural shortening of the tube axis is balanced by a corresponding increase of the average cross-section ('fatning' process). Evidently, this process of energy reduction comes to a halt when different parts of the tube come into contact with each other, at which stage topological constraints put a barrier to any further relaxation of magnetic tension and the relaxation comes to a halt (see figure 5). Positive lower bounds on magnetic energy minimization must therefore exist (although these may not be uniquely defined), according to the specific link or knot topology. A result that entails both magnetic energy and topology of $\mathcal{L}_{m}$ is given by the following:

Theorem 5 (Freedman, 1988; Moffatt, 1992). Let $\mathcal{L}_{m}$ be a magnetic link in $\mathcal{D}_{1}$. Then there exists a positive lower bound such that

$$
\lim _{t \rightarrow \infty} E_{m}(t)=E_{m, \min }>0
$$


and

$$
E_{m}\left(\mathcal{L}_{m}\right) \geq q_{0}\left|H\left(\mathcal{L}_{m}\right)\right|,
$$

where $q_{0}$ is a non-zero positive constant independent of $\mathcal{L}_{m}$.

This means that for non-trivial topologies $\left(\mathcal{L}_{m}\right.$ is not homeomorphic to unlinked tori) we can show that even if $H\left(\mathcal{L}_{m}\right)=0$ (as in the case shown in figure 4), then there exist positive lower bounds on $E_{m}(t)$. End states (corresponding to ground energy levels) are attained when different parts of flux-tubes come into contact with each other; when this happens, tangential discontinuities in the $\mathbf{B}$-field may arise: in this case fluid flow maps becomes ill-defined (contact regions are images of pathological flow maps) and new initial conditions for the fluid flow are needed.

If $\mathcal{L}_{m} \equiv \mathcal{K}_{m}$ is a single knotted magnetic flux-tube, then Moffatt (1990) has shown (on dimensional grounds) that the energy of the end state can be written as

$$
E_{m, \min }\left(\mathcal{K}_{m}\right)=m(h)\left[\Phi\left(\mathcal{K}_{m}\right)\right]^{2} V\left[\left(\mathcal{K}_{m}\right)\right]^{1 / 3},
$$

where $m$ is a positive real number, determined only by the knot topology, and $h$ is a twist parameter. However, as $t \rightarrow \infty$ the knot end state is not uniquely determined. Evidently the energy re-distribution influences the magnetic relaxation process; for a given knot a spectrum of potentially different asymptotic end states is expected. The knot energy spectrum would be given by $\left\{m_{0}, m_{1}, m_{2}, \ldots\right\}$ ( $m_{0}$ being the ground state energy), where a high value of $m_{0}$ indicates high knot complexity. Numerical results obtained by Chui \& Moffatt (1995) seem to indicate that indeed torus knots $\mathcal{K}_{m} \equiv \mathcal{T}_{p, q}$ ( $p, q$ co-prime integers) appear to be characterized by quantised energy levels, with energy growing with crossing numbers. Additional calculations show that the two magnetic torus knot isotopes, given for example by $\mathcal{T}_{2,3}$ and $\mathcal{T}_{3,2}$, can both attain the ground state energy level if the internal twist of field lines is altered (which corresponds to a change in $h$, hence in the framing of $\mathcal{T}_{p, q}$ and in the self-linking number of the knot).

Local equilibria are likely to depend on both topology and geometric properties of the magnetic field distribution. Whether global minima depend solely on topology, however, is still an open question. Lower bounds on (minimum) magnetic energy of knotted flux-tubes were found by Freedman \& He (1991). It can be shown that

$$
E_{m, \min }\left(\mathcal{K}_{m}\right) \geq \frac{\left[\Phi\left(\mathcal{K}_{m}\right)\right]^{3 / 2}\left[a c\left(\mathcal{K}_{m}\right)\right]^{3 / 4}}{\pi^{1 / 4}\left[V\left(\mathcal{K}_{m}\right)\right]^{1 / 3}},
$$

where

$$
a c\left(\mathcal{K}_{m}\right)=<n_{+}(\nu)+n_{-}(\nu)>,
$$

is the asymptotic crossing number of the knot type (Freedman \& He conjecture that $a c\left(\mathcal{K}_{m}\right)=c\left(\mathcal{K}_{m}\right) \equiv c_{K}$, where $c_{K}$ is the topological crossing number of the knot type, i.e. the number of crossings in the minimal knot diagram).

Magnetic knots can be isotoped to closed magnetic braids (in the conjugacy class of the knot type) by application of Markov's theorem (Birman, 1976; Hansen, 1989) and action of local fluid flows on tube strands. In absence of background flows (fluid at rest) we can show that magnetic knots with points of inflexions, i.e. points at which the curvature of the tube axis vanishes, evolve to inflexion-free braids and then to minimal 
braids. By using first principles of ideal magnetohydrodynamics (MHD) (no viscous or resistive effects present) inflexional magnetic knots remove inflexion points through a generic (natural) deformation driven by the Lorentz force. We have:

Theorem 6 (Ricca, 1996a). Let $\varphi_{t}(\mathcal{K}) \equiv \mathcal{K}_{t}$ be the embedding of a magnetic knot in $\mathcal{D}$. If $\mathcal{K}_{t_{1}}$ has a finite number of inflexion points in isolation present at time $t_{1}$, then $\mathcal{K}_{t_{1}}$ is naturally isotoped to the inflexion-free knot $\mathcal{K}_{t_{2}}\left(t_{2}>t_{1}\right)$, i.e. $\varphi: \mathcal{K}_{t_{1}} \mapsto \mathcal{K}_{t_{2}}$, for some $\left\{t_{1}, t_{2}\right\} \in I, t_{2}>t_{1}$.
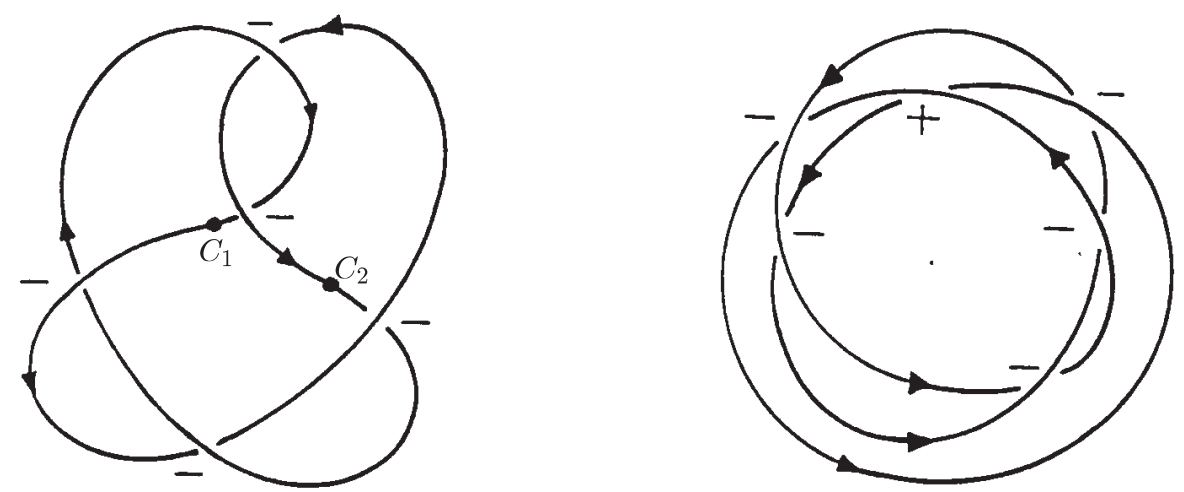

Fig. 6. The knot $K_{5,2}$ on the left is shown in standard projection with minimum number of crossings $\left(c_{K}=5\right)$ and two (apparent) points of inflexion $\left(C_{1}\right.$ and $\left.C_{2}\right)$. The same knot can be isotoped to an inflexion-free braid configuration (on the right-hand-side of the figure) with number of crossings $c_{B} \geq 6$.

This result shows that there is a natural connection between magnetic knots and minimal braids. Since magnetic relaxation induces a shortening flow that shrinks the tube axis and also removes inflexions, magnetic knots evolve quite naturally to closed braid configurations. Depending on the knot geometry, this deformation may yield to the appearance of a higher number of crossings in the resulting inflexion-free braid (see figure 6): if $c_{K}$ denotes the topological crossing number for the knot type $\mathcal{K}_{t_{1}}$, then the corresponding closed braid $\mathcal{B} \equiv \mathcal{K}_{t_{2}} \sim \mathcal{K}_{t_{1}}$ has number of crossings $c_{B}$ that according to Vogel (1990) and Traczyk (1995) is bounded by

$$
c_{K} \leq c_{B} \leq c_{K}+\left(c_{K}-1\right)\left(c_{K}-2\right) .
$$

Since inflexional configurations represent disequilibria for magnetic knots, then inflexional knots evolve to braided configurations at lower energy levels. The tendency of knotted magnetic flux-tubes to remove inflexions and relax to lower energy states indicate that magnetic braids (and minimal braids in particular) are favourite candidates for low energy states.

Finally, let us consider an open braid $\mathcal{B}_{m}$ of $N$ components (corresponding to $N$ equal magnetic flux-tubes). The braid is given by $N$-strings placed between two planes $z=0$ and $z=L$, embedded in an inviscid, cylindrical sub-domain $\mathcal{D}_{0} \equiv\left(x^{2}+y^{2} \leq R\right) \otimes(0 \leq$ $z \leq L) \subset \mathcal{D}\left(R\right.$ and $L$ are finite quantities). For a magnetic field given by $\mathbf{B}=B_{z}(\mathbf{b}+\mathbf{k})$, 
where $B_{z}$ is constant and $\mathbf{b}=[(d x / d z) \mathbf{i},(d y / d z) \mathbf{j}]$ (with $\mathbf{i}, \mathbf{j}, \mathbf{k}$ standard Cartesian unit vectors), the free magnetic energy of the braid is given by

$$
E_{m}\left(\mathcal{B}_{m}\right)=\frac{B_{z}^{2}}{8 \pi} \int_{\cup_{i} \mathcal{B}_{m}\left(T_{i}\right)}|\mathbf{b}|^{2} d V_{i}, \quad \text { (Gaussian units). }
$$

Assuming that the $N$ tubes have equal magnetic flux given by $\Phi=\pi R^{2} B_{z} / N$, we can prove the following result:

Theorem 7 (Berger, 1993). Let $\mathcal{B}_{m}$ be a magnetic braid in $\mathcal{D}$. Then there exists a positive lower bound for the magnetic energy given by

$$
E_{m}\left(\mathcal{B}_{m}\right) \geq 9.06 \times 10^{-2}\left[c_{\text {min }}\left(\mathcal{B}_{m}\right)\right]^{2} \frac{\Phi^{2}}{N^{2} L}, \quad \text { (Gaussian units) },
$$

where $c_{\text {min }}\left(\mathcal{B}_{m}\right)$ is the minimum crossing number of the $N$-braid.

Moreover, if the flux-tubes are subject to random motion about each other, with typical velocity $\mathbf{u}$ and average distance between foot-points $d$, then Berger (1993) has shown that

$$
c_{m i n, t}\left(\varphi_{t}\left(\mathcal{B}_{m}\right)\right)=\gamma \frac{|\mathbf{u}| t}{d} N^{3 / 2},
$$

where $\gamma$ is an efficiency parameter. This result has important implications in astrophysical contexts, where energy estimates of braided magnetic structures provide useful information for the study of production and dissipation of magnetic energy in solar coronal loops.

7. Vortex knots and integrability. Vortex knots $\mathcal{L}_{v}$ are the magnetic analog of knotted magnetic flux-tubes and are defined simply by replacing the $\mathbf{B}$-field with the $\omega$-field (vorticity). Under the Euler equations vortex dynamics is governed by the BiotSavart integral, that is a global functional of vorticity $\omega$. This integral gives the propagation velocity of the vortex (Batchelor, 1967). If the vortex filament is very thin (i.e. with radius of the cross-section small compared to the radius of curvature of the tube axis) and the interactions of different parts of the vortex are neglected, then the Biot-Savart integral is replaced by an asymptotic theory and the motion of the vortex is approximated by simple local laws ('localized induction approximation' laws, or LIA for short). Under LIA we have a family of dynamical laws of increasing complexity, depending on the degree of approximation of the physical model. Under these laws we may have a hierarchy of soliton equations (such as the Non-Linear Schrödinger equation or the Korteweg-de Vries equation). These equations are completely integrable and have a countably infinite number of polynomial invariants that can be interpreted as global geometric functionals (Ricca, 1990; Langer \& Perline 1991; Moffatt \& Ricca, 1991). For example, under a zeroth-order LIA, the motion of a single vortex knot $\mathcal{L}_{v}(T) \equiv \mathcal{K}_{v}(T)$ is governed by a very simple law, that after appropriate re-scaling of time takes the form

$$
\mathbf{u}=\dot{\mathbf{X}}=\mathbf{X}^{\prime} \times \mathbf{X}^{\prime \prime}=c \mathbf{b}
$$

where the time-derivative is denoted by a dot and primes denote derivatives with respect to arc-length of the tube axis; $c$ and $\mathbf{b}$ are the curvature and the unit binormal of the axis. Hence, according to (34), the velocity induces a 'binormal flow' on $\mathcal{K}_{v}$. 
Existence, steadiness and stability of knotted solutions to Euler's equations have been conjectured since the original works of Kelvin (1869), but so far only few analytical results have been put forward. In the LIA context, though, some progress has been made. An important result is the following:

Theorem 8 (Kida, 1981). Let $\mathcal{K}_{v}(T)$ denote the embedding of a vortex knot in $\mathcal{D}$. If $\mathcal{K}_{v}$ evolves under the zeroth-order LIA, then there exists a class of steady solutions in the shape of torus knots $\mathcal{K}_{v} \equiv \mathcal{T}_{p, q}$, where $p>1$ and $q>1$ are co-prime integers.

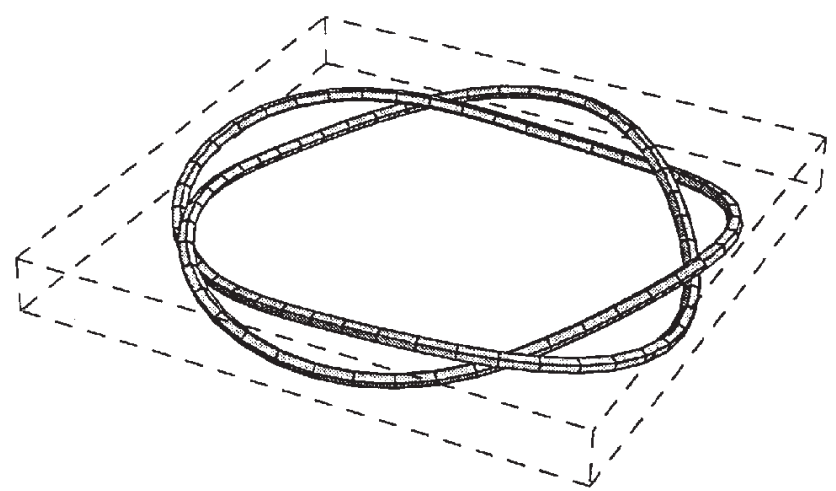

Fig. 7. Vortex tube in the shape of 'small-amplitude' torus knot $\mathcal{T}_{2,5}$. This represents a stable solution to the zeroth-order LIA (eq. 34) (Ricca, 1993; 1995a).

These correspond to fully non-linear solutions to LIA. In the linear régime, 'smallamplitude' torus knots and cable-knots have been found by Keener (1990) and Ricca (1993; 1995a). A linear stability analysis based on explicit analytic expressions for torus knot solutions under LIA (see figure 7 ) leads to the following result:

Theorem 9 (Ricca, 1993; 1995a). Let $\mathcal{T}_{p, q}(p>1$ and $q>1$ co-prime integers $)$ be the embedding of a 'small-amplitude' vortex torus knot in $\mathcal{D}$, solution to the zeroth-order LIA. Under linear perturbations, $\mathcal{T}_{p, q}$ is stable for $p<q$, and unstable for $p>q$.

This means that $\mathcal{T}_{p, q}$-vortex knots with $p / q>1$ are dynamically unstable and evolve to different configurations. Remember that under LIA reconnections are not allowed (this is an artificial requirement of the asymptotic analysis); consequently, $\mathcal{T}_{p, q}$ knots (with $p / q>1)$ evolve towards a singularity event. On the other hand, for given $(p, q)$ we have that $\mathcal{T}_{p, q} \sim \mathcal{T}_{q, p}$. Stability characteristics will therefore select those long-lived structures from the family of topologically equivalent configurations.

Under the Euler equations the topology of vorticity is preserved, but the enstrophy of the associated flow, defined by

$$
\Omega(t)=\Omega\left(\varphi_{t}\left(\mathcal{L}_{v}\right)\right)=\int_{\cup_{i} \mathcal{L}_{v}\left(T_{i}\right)}|\omega|^{2} d V_{i}
$$

is not conserved. It is obvious that there can be no theoretical upper bound on the enstrophy, because natural evolution governed by the Euler equations is generally such as to increase enstrophy without limit when the effects of viscosity are ignored (Batchelor, 
1967). As in the case of magnetic relaxation, we might ask whether, given the topology of the vorticity field, there is any lower bound to the enstrophy, under the action of volumepreserving diffeomorphisms. By simple application of the Schwarz inequality Moffatt (1969) showed that when the kinetic helicity (where $\mathbf{u} \cdot \omega$ is now the helicity density) of a flow is non-zero, in ideal conditions the enstrophy is bounded below. A lower bound may be attained by the same technique used in the context of magnetic relaxation, since magnetostatic equilibria correspond to steady solutions of Euler's equations. For a collection of vortex links $\left(\mathcal{L}_{v}\right)$, each tube having different flux $\Phi_{i}$ but equal volume $V$, it can be shown (Moffatt \& Ricca, 1991) that enstrophy takes the form

$$
\Omega\left(\mathcal{L}_{v}\right)=\sum_{i, j} m_{i j} \Phi_{i} \Phi_{j} V^{-1 / 3}
$$

where $m_{i j}$ is a real, symmetric, positive-definite matrix. Under frozen-field deformation, this matrix varies, and, for given $\Phi_{i}, \Phi_{j}$, there is a minimising configuration. However, $m_{i j}$ in the minimum enstrophy state is a function of the ratio $\Phi_{i} / \Phi_{j}$. This may be seen explicitly for the Whitehead link, for which $m_{11}$ is non-zero if $\Phi_{2}$ is non-zero.

8. Charged knots and elastic strings. The influence of knot topology on minimum energy levels can be studied with electrically charged knots and elastic strings. Charged links (knots) $\mathcal{L}_{q}$ are made of flexible and inextensible loops of wire carrying an electrical charge $Q . \quad \mathcal{L}_{q}$ is embedded in a non-conducting, non-dielectric, viscous medium (like honey). The so-called 'honey jar problem' (from Freedman \& He, 1992) consists of finding minimum energy configurations attained by $\mathcal{L}_{q}$ due to the action of the repulsive Coulomb forces and the dissipative forces of fluid viscosity. The charged knot moves in the fluid driven by a local force that in first approximation is given by $\mathbf{F} \propto-c \mathbf{n}$, where $c$ is the curvature of the axis and $\mathbf{n}$ is the unit normal: because of the minus sign, the charged knot is subject to a 'straightening flow' action.

Different knot models have been proposed; the physical knot can be modelled by an infinitely thin filament, a hollow tube or a solid tube, and it can be either a perfect conductor or made of infinitely resistive material (with zero conductivity). A discussion of the physical implications that these different models have on the relaxation mechanism is given by Lomonaco (1995).

Definition 9. The potential energy associated with $\mathcal{L}_{q}$ is defined by

$$
E_{q}=\frac{1}{2} \oint_{\mathcal{L}_{q}} \oint_{\mathcal{L}_{q}} \frac{\lambda(s) \lambda\left(s^{*}\right)}{\left|\mathbf{X}(s)-\mathbf{X}\left(s^{*}\right)\right|} d s d s^{*},
$$

where $\mathbf{X}(s)$ denotes the filament (or tube) axis, $s$ the arc-length and $\lambda(s)$ the charge density function.

As $s \rightarrow s^{*}$ the integral becomes divergent and there are analytical difficulties to describe the relaxation process. In general, these difficulties are overcome by using techniques of de-singularisation based on asymptotics, 'cut-off' methods or analytical renormalisation of singularities.

Potential asymptotic theories of thin tube geometries have been put forward since the original work of Levi-Civita (1909) (for a review of Levi-Civita's asymptotic potential 
theory see Ricca, 1996b). Techniques based on appropriate cut-off of the singularity point have been used in numerical analysis (see, for example, Fukuhara, 1988), where cut-off lengths are introduced through the numerical discretisation algorithm, while artificial re-normalisation of the Green function is a more modern idea. Birman and Lomonaco (see Lomonaco, 1995 and references therein), for example, replace $\left|\mathbf{X}(s)-\mathbf{X}\left(s^{*}\right)\right|$ with $\left(\left|\mathbf{X}(s)-\mathbf{X}\left(s^{*}\right)\right|+\epsilon\right)$ and study the relaxation in the limit for small $\epsilon$.

Alternatively, we can define re-normalised 'energy' functionals as follows. For simplicity, let us consider links (knots) with constant charge $\lambda(s)=1$. According to Freedman \& He (1992; see also Freedman, He \& Wang, 1994), we have

Definition 10. The $\alpha$-re-normalised energy (pseudo-energy) of the link $\mathcal{L}_{q}, E_{\alpha}\left(\mathcal{L}_{q}\right)$ $(\alpha \geq 1)$, is defined by

$$
E_{\alpha}\left(\mathcal{L}_{q}\right)=\oint_{\mathcal{L}_{q}} \oint_{\mathcal{L}_{q}}\left(\frac{1}{\left|\mathbf{X}(s)-\mathbf{X}\left(s^{*}\right)\right|^{\alpha}}-\frac{1}{\left[D\left(\mathbf{X}(s), \mathbf{X}\left(s^{*}\right)\right)\right]^{\alpha}}\right) d s d s^{*},
$$

where $D\left(\mathbf{X}(s), \mathbf{X}\left(s^{*}\right)\right)$ measures the length of the arc $s s^{*}$ on the curve $\mathbf{X}(s)$.

It should be noted that only the energy defined by (37) has physical meaning. Similar re-normalisations are used by O'Hara (1991; see also Simon, 1994). For charged knots $\mathcal{L}_{q} \equiv \mathcal{K}_{q}$, Freedman \& He (1992) show that the topological crossing number $c_{K}$ is bounded from above by the 2-re-normalised energy ('Möbius' energy), according to the formula

$$
E_{2}\left(\mathcal{K}_{q}\right) \geq \frac{12 \pi}{11}\left(c_{K}-\frac{1}{\pi}\right)
$$

which poses an upper limit on the number of topologically distinct knot types below a given threshold of energy level. Examples of charged torus knots that are critical for this pseudo-energy are given by Kim \& Kusner (1993).

Other energy functionals have physical meaning. For instance, consider structures with elastic properties given by the elasticity and the rigidity characteristics of some material. Flexible elastic strings, for example, deform to reduce elastic tension and internal stress in a way similar to the behaviour of magnetic flux-tubes. Knotted strings $\mathcal{K}_{e}$ modelled by thin, inextensible, elastic tubes can be studied by applying relaxation techniques to elastic energy functionals. In the context of linear elasticity, the elastic energy functional is given by

$$
E_{e}\left(\mathcal{K}_{e}\right)=\oint_{\mathcal{K}_{e}}\left(\frac{C_{b}}{2}[c(s)]^{2}+\frac{2 \pi^{2} C_{t}}{L^{2}}[T w(s)]^{2}\right) d s
$$

where $C_{b}$ and $C_{t}$ are the coefficients of bending rigidity and torsional rigidity, $c(s)$ is the curvature of the axis, and $T w$ and $L$ denote total twist and length of the string. Here, for simplicity, curvature and twist of the reference (or natural) configuration are set to zero.

Many studies of elastic relaxation of twisted flexible strings are based on the energy functional (40) and on conservation of (Călugăreanu-White) linking number (see, for example, Tanaka \& Takahashi, 1985; Wadati \& Tsuru, 1986; Schlick \& Olson, 1992; Ricca, 1995b; see also the results collected by Sumners, 1992). At different linking number values, writhe and twist contribute differently to the loop configuration, leading to the development of supercoiling at high writhing number. The exchange of twist and writhe 

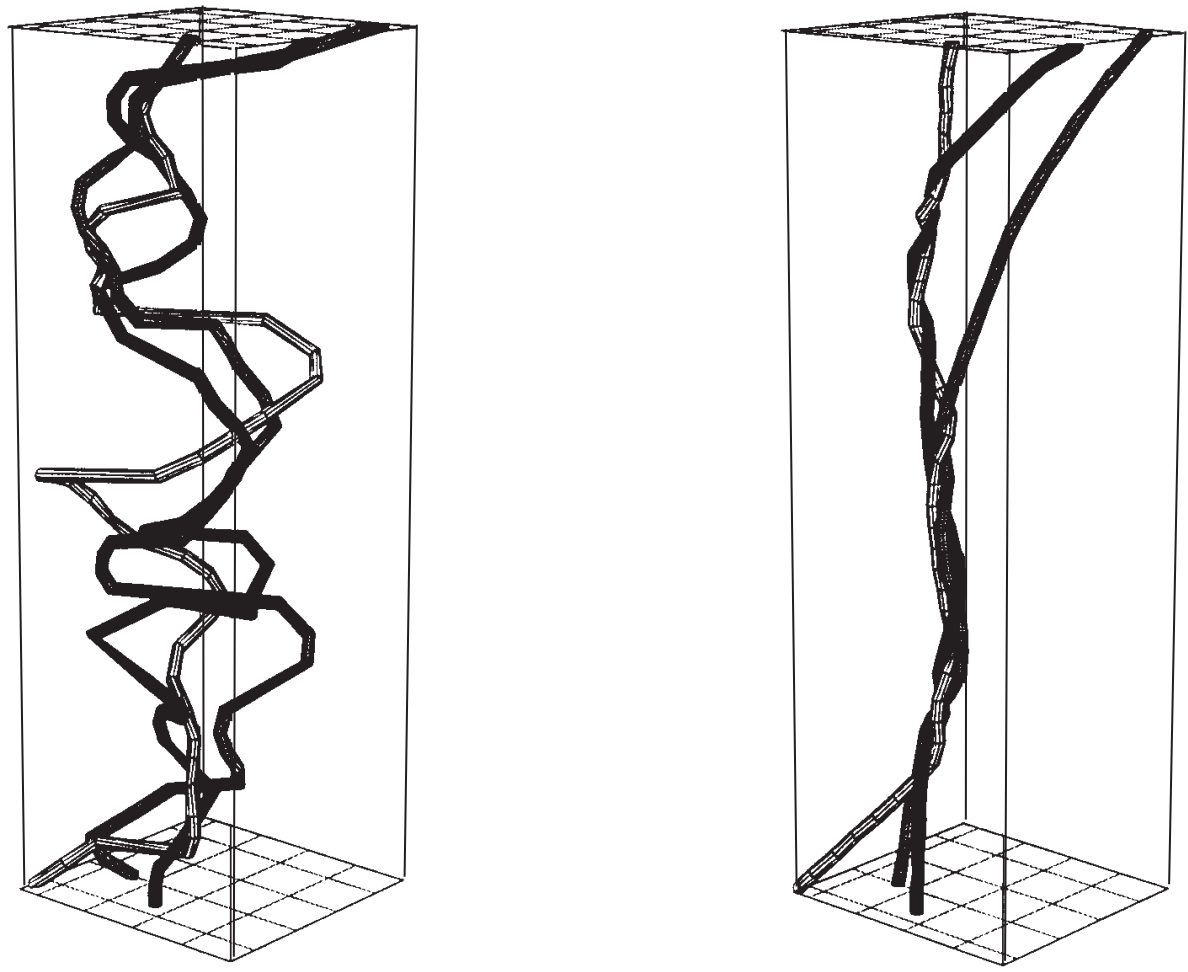

Fig. 8. Example of elastic relaxation of a 3-component braid of elastic strings. The system on the left, with high elastic energy, evolves from a randomly generated initial state to a relaxed low energy end-state (on the right) under minimisation of a curvature force (Schilham, 1995).

performed naturally on the string through a sequence of Reidemeister moves (a process that generally involves the appearance of points of inflexions) and the effects on the energy levels have been investigated by Ricca (1995b). Critical energy levels are found to depend strongly on specific linking differences and elastic characteristics of $\mathcal{K}_{e}$. Moreover, it can be shown that for very high values of specific linking difference (i.e. high superhelicity) the writhing number of the supercoil state tends to a limit (independent of the elastic charateristics of the string) that gives upper bounds on the bending energy $E_{e, b}\left(\mathcal{K}_{e}\right)$ (given by the first contribution in the r.h.s. of eq. 40). Since this energy is given by the total square curvature, this means that there are upper bounds on the total square curvature too.

A fundamental theorem of Milnor (1950) relates global geometry and knot topology. Let us first recall the following definition (Rolfsen, 1976):

Definition 11. The bridge index $\beta(K)$ of a knot is the minimum number of maximal bridges (over-passes of the knot diagram that do not contain under-crossing points) over all knot diagrams. By convention $\beta$ (unknot $)=1$.

The bridge index is an invariant of the knot. Milnor's theorem states that: 
TheOREM 10 (Milnor, 1950). For a simple, closed and smooth knot $K$ of total curvature $\kappa(K)$ given by

$$
\kappa(K)=\oint_{K} c(s) d s
$$

and bridge index $\beta(K)$, we have

$$
\kappa(K) \geq 2 \pi \beta(K)
$$

Moreover:

Corollary 11. If $\kappa(K)<4 \pi$, then $K$ is the unknot.

We can relate elastic energy levels with knot topology by a simple application of Schwarz inequality; denoting by $\hat{E}_{e, b}\left(\mathcal{K}_{e}\right)$ the bending energy divided by $C_{b} / 2 L\left(C_{b}\right.$ and $L$ are assumed constant), we have

$$
\hat{E}_{e, b}\left(\mathcal{K}_{e}\right)=L \oint_{\mathcal{K}_{e}}[c(s)]^{2} d s \geq\left[\kappa\left(\mathcal{K}_{e}\right)\right]^{2} \geq 4 \pi^{2}\left[\beta\left(\mathcal{K}_{e}\right)\right]^{2},
$$

$\left(\beta\left(\mathcal{K}_{e}\right) \equiv \beta(K)\right)$ which gives (similarly to eq. 39 ), upper bounds on the admissible number of distinct knot types below a given threshold of bending energy.

Suppose now that $\mathcal{K}_{e}$ is deformed to lie almost entirely on a plane, (or on a sphere), allowing just small indentations at crossing points due to the over-passes and under-passes of the strands. If $\mathcal{K}_{e}$ has no intrinsic twist we can show that in the limit of vanishing cross-section and small indentations the total torsion of the string axis tends to zero and the total energy becomes bounded from below

$$
\hat{E}_{e}\left(\mathcal{K}_{e}\right) \geq 4 \pi^{2}\left[\beta\left(\mathcal{K}_{e}\right)\right]^{2} .
$$

Similar estimates based on knot topology and energy relaxation are very useful in a variety of model problems (see figure 8).

Finally, we want to mention a result due to Langer \& Singer $(1984 ; 1985)$ on elastic knots under the action of a 'straightening flow':

TheOREM 12 (Langer \& Singer, 1984; 1985). There exists a countably infinite family of (similarity classes of) closed non-planar elastic curves in $\mathbb{R}^{3}$. All such elasticae are embedded and lie on embedded tori of revolution. Infinitely many of these are knotted and the knot types which thus occur are precisely the $\mathcal{T}_{p, q}$ torus knots satisfying $p>2 q$. The integers $p, q$ determine the elasticae uniquely (up to similarity).

Experiments on the elastic relaxation of closed, knotted loops made of springy wire show that there are at least three points of self-contact (counting multiplicity) to have stable configurations in equilibrium. When this happens, impressive symmetries do arise.

9. Conclusions. Fluid mechanics provides a natural context for applications of ideas and results from knot theory. In this paper we have presented and discussed some of these applications that are part of the most recent developments in topological fluid mechanics (TFM). This is a growing discipline that is already becoming diversified in many subfields. It is therefore difficult, if not impossible, to present a balanced overview of the state-of-art of the subject. The choice of the topics discussed here has been deliberately 
restricted to those areas that are best known to the author and that involve a combination of ideal fluid mechanics and topological concepts. A brief overview of other related aspects has been given in $\S 1$.

The concept of topological equivalence of fluid flows and structures has been discussed in $\S 2$, and knotted and linked flux-tubes have been defined through basic working definitions in $\S 3$. A fluid mechanical interpretation of Reidemeister's moves in terms of fluid flows has been discussed in $\S 4$. In the context of the Euler equations, a theorem of Lichtenstein (1925) concerning the isotopic evolution of vortex structures has been presented and discussed in the TFM context for the first time. Proof of the theorem can be found in the original paper by Lichtenstein and in the XII chapter of Villat's book (1930, pp. 256-269), and is based on the Cauchy equations for a divergence-free velocity field. In this context Lichtenstein's theorem may be considered one of the first examples of topological interpretation of fluid mechanics actions of fluid flows.

The topological interpretation of the helicity of a fluid flow in terms of linking numbers has been discussed in $\S 5$. An overview of results on magnetic relaxation of linked, knotted and braided structures has been presented in $\S 6$. Interesting relationships between energy levels and topological information have been reviewed together with a discussion of results on knotted magnetic flux-tubes and corresponding braid configurations. In $\S 7$ we have examined relationships between integrability and existence and stability of vortex filaments in the shape of torus knots in the context of the Euler equations and under certain approximations. Finally, in $\S 8$, we have discussed a number of results concerning electrically charged knots in a viscous fluid, elastic relaxation of loops and braided strings. New bounds for elastic energy levels based on global geometric quantities and topological information have been presented.

Many other results have been left out and we apologise for this. Our hope is that this review will at least convey the sense of beauty and excitement given by the fascinating progress of topological fluid mechanics.

\section{References}

[1] H. Alfvén, Cosmic Electrodynamics, Oxford University Press, 1950.

[2] H. Aref and I. Zawadzki, Linking of vortex rings, Nature 354 (1991), 50-53.

[3] V.I. Arnold, The asymptotic Hopf invariant and its applications, in: Summer School in Diff. Eqs., Proc. Acad. Sci. Armenian S.S.R., Erevan, 1974, 229-256 (in Russian).

[4] V.I. Arnold and B.A. Khesin, Topological Methods in Hydrodynamics, Ann. Rev. Fluid Mech. 24 (1992), 145-166.

[5] G.K. Batchelor, An Introduction to Fluid Dynamics, Cambridge University Press, 1967.

[6] T. Bedford and J. Swift (ed.), New Directions in Dynamical Systems, Cambridge University Press, 1988.

[7] M.A. Berger, Third order link invariants, J. Phys. A: Math. \& Gen. 23 (1990), 2787-2793.

[8] M.A. Berger, Third order braid invariants, J. Phys. A: Math. \& Gen. 24 (1991), 4027-4036.

[9] M.A. Berger, Energy-crossing number relations for braided magnetic fields, Phys. Rev. Lett. 70 (1993), 705-708. 
[10] M.A. Berger and G.B. Field, The topological properties of magnetic helicity, J. Fluid Mech. 147 (1984), 133-148.

[11] J.S. Birman, Braids, links and mapping class group, Annals of Math. Studies 82, Princeton University Press, 1976.

[12] J.S. Birman and R.F. Williams, Knotted periodic orbits in dynamical systems I: Lorenz's equations, Topology 22 (1983), 47-82.

[13] O.N. Boratav, R.B. Pelz and N.J. Zabusky, Reconnection in orthogonally interacting vortex tubes: direct numerical simulations and quantifications, Phys. Fluids A 4 (1992), 581-605.

[14] R.J. Bray, L.E. Cram, C.J. Durrant and R.E. Loughhead, Plasma Loops in the Solar Corona, Cambridge University Press, 1991.

[15] G. Călugăreanu, On isotopy classes of three-dimensional knots and their invariants, Czechoslovak Math. J. T11 (1961), 588-625 (in French).

[16] A.Y.K. Chui and H.K. Moffatt, The energy and helicity of knotted magnetic flux-tubes, Proc. Roy. Soc. Lond. A 451 (1995), 609-629.

[17] N.W. Evans and M.A. Berger, A hierarchy of linking integrals, in: Topological Aspects of the Dynamics of Fluids and Plasmas, H.K. Moffatt et al. (ed.), Kluwer, Dordrecht, The Netherlands, 1992, 237-248.

[18] M.H. Freedman, A note on topology and magnetic energy in incompressible perfectly conducting fluids, J. Fluid Mech. 194 (1988), 549-551.

[19] M.H. Freedman and M.A. Berger, Combinatorial relaxation of magnetic fields, Geophys. Astrophys. Fluid Dynamics 73 (1993), 91-96.

[20] M.H. Freedman and Z.-X. He, Divergence-free fields: energy and asymptotic crossing number, Ann. Math. 134 (1991), 189-229.

[21] M.H. Freedman and Z.-X. He, Research announcement on the "energy" of knots, in: Topological Aspects of the Dynamics of Fluids and Plasmas, H.K. Moffatt et al. (ed.), Kluwer, Dordrecht, The Netherlands, 1992, 219-222.

[22] M.H. Freedman, Z.-X. He and Z. Wang, Möbius energy of knots and unknots, Ann. Math. 139 (1994), 1-50.

[23] S. Fukuhara, Energy of a knot, in: A Fete of Topology: Papers Dedicated to Itiro Tamura, Y.T. Matsumoto and S. Morita (ed.), Academic Press, New York, 1988, 443451.

[24] F.B. Fuller, The writhing number of a space curve, Proc. Nat. Acad. Sci. U.S.A. 68 (1971), 815-819.

[25] C.F. Gauss, Works, Königliche Gesellschaft der Wissenschaften zu Göttingen, 1877 (in German).

[26] V.L. Hansen, Braids and Coverings, London Math. Soc. 18, Cambridge University Press, 1989.

[27] H. Helmholtz, On integrals of the hydrodynamical equations, which express vortex motion, J. Reine Angew. Math. 55 (1858), 25-55 (in German).

[28] P.J. Holmes and R.F. Williams, Knotted periodic orbits in suspensions of Smale's horseshoe: torus knots and bifurcation sequences, Arch. Rat. Mech. Anal. 90 (1985), 115-194.

[29] J. Jiménez (ed.), The Global Geometry of Turbulence, NATO ASI B 268, Plenum Press, New York, 1991.

[30] B.B. Kadomtsev, Magnetic field line reconnection, Rep. Prog. Phys. 50 (1987), 115-143.

[31] L.H. Kauffman, On Knots, Annals Study 115, Princeton University Press, 1987.

[32] L.H. Kauffman, [a] Knots and Applications, World Scientific, Singapore, 1995. 
[33] L.H. Kauffman, [b] The interface of Knots and Physics, Proc. Symp. Appl. Maths. 51, Am. Math. Soc., 1995.

[34] J.P. Keener, Knotted vortex filaments in an ideal fluid, J. Fluid Mech. 211 (1990), 629-651.

[35] Lord Kelvin (W.T. Thomson), On vortex motion, Trans. Roy. Soc. Edinb. 25 (1869), $217-260$

[36] Lord Kelvin (W.T. Thomson), Vortex statics, Proc. Roy. Soc. Edinb. ss. 1875-76 (1875), 115-128.

[37] B.A. Khesin and Yu.V. Chekanov, Invariants of the Euler equations for ideal or barotropic hydrodynamics and superconductivity in D dimensions, Physica D 40 (1989), 119131.

[38] S. Kida, A vortex filament moving without change of form, J. Fluid Mech. 112 (1981), 397-409.

[39] D. Kim and R. Kusner, Torus knots extremizing the conformal energy, Experimental Math. 2 (1993), 1-9.

[40] E.A. Kuznetsov and A.V. Mikhailov, On the topological meaning of canonical Clebsch variables, Phys. Lett. 77 A (1980), 37-38.

[41] J. Langer and R. Perline, Poisson geometry of the filament equation, J. Nonlinear Sci. 1 (1991), 71-93.

[42] J. Langer and D.A. Singer, Knotted elastic curves in $\mathbb{R}^{3}$, J. London Math. Soc. 30 (1984), 512-520.

[43] J. Langer and D.A. Singer, Curve straightening and a minimax argument for closed elastic curves, Topology 24 (1985), 75-88.

[44] T. Levi-Civita, On mechanical actions due to a filiform flux of electricity, Rend. R. Acc. Lincei 18 (1909), 41-50 (in Italian).

[45] L. Lichtenstein, On some existence problems of the hydrodynamics of homogeneous and incompressible, inviscid fluid and the vortex laws of Helmholtz, Math. Zeit. 23 (1925), 89-154 (in German).

[46] S.J. Lomonaco, The modern legacies of Thomson's atomic vortex theory in classical electrodynamics, in: The interface of Knots and Physics, Proc. Symp. Appl. Maths. 51, Am. Math. Soc., 1995.

[47] H.J. Lugt, Vortex Flow in Nature and Technology, J. Wiley \& Sons, New York, 1983.

[48] J. Marsden and A. Weinstein, Coadjoint orbits, vortices, and Clebsch variables for incompressible fluids, Physica D 7 (1983), 305-323.

[49] J.C. Maxwell, A Treatise on Electricity and Magnetism, MacMillan \& Co., Oxford, 1873.

[50] K.R. Meyer and D.G. Saari, Hamiltonian Dynamical Systems, Contemp. Math. 81, New York, 1988.

[51] J. Milnor, On the total curvature of knots, Ann. Math. 52 (1950), 248-257.

[52] H.K. Moffatt, The degree of knottedness of tangled vortex lines, J. Fluid Mech. 35 (1969), $117-129$.

[53] H.K. Moffatt, The energy spectrum of knots and links, Nature 347 (1990), 367-369.

[54] H.K. Moffatt, Relaxation under topological constraints, in: Topological Aspects of the Dynamics of Fluids and Plasmas, H.K. Moffatt et al. (ed.), Kluwer, Dordrecht, The Netherlands, 1992, 3-28.

[55] H.K. Moffatt and R.L. Ricca, Interpretation of invariants of the Betchov-Da Rios equations and of the Euler equations, in: The Global Geometry of Turbulence, J. Jiménez (ed.), NATO ASI B 268, Plenum Press, New York, 1991, 257-264. 
[56] H.K. Moffatt and R.L. Ricca, Helicity and the Călugăreanu invariant, Proc. R. Soc. Lond. A 439 (1992), 411-429.

[57] H.K. Moffatt and A. Tsinober (ed.), Topological Fluid Mechanics, Cambridge University Press, 1990.

[58] H.K. Moffatt, G.M. Zaslavsky, P. Comte and M. Tabor (ed.), Topological Aspects of the Dynamics of Fluids and Plasmas, Kluwer, Dordrecht, The Netherlands, 1992.

[59] J.-J. Moreau, Constants of a vortex filament in a barotropic perfect fluid, C. R. Acad. Sci. Paris 2 (1961), 2810-2812 (in French).

[60] J. O'Hara, Energy of a knot, Topology 30 (1991), 241-247.

[61] R.L. Ricca, Global geometric invariants of the Da Rios-Betchov equations, Internatl. Symp. on Cont. Mech., USSR Acad. Sci., Perm-Moscow, 1990.

[62] R.L. Ricca, Torus knots and polynomial invariants for a class of soliton equations, Chaos 3 (1993), 83-91 [Erratum: Chaos 5 (1995), 346].

[63] R.L. Ricca, [a] Geometric and topological aspects of vortex filament dynamics under LIA, in: Small-Scale Structures in Three-Dimensional Hydro and Magnetohydrodynamics Turbulence, M. Meneguzzi et al. (ed.), Lecture notes in Physics 462, Springer-Verlag, 1995, 99-104.

[64] R.L. Ricca, [b] The energy spectrum of a twisted flexible string under elastic relaxation, J. Physics A: Math. \& Gen. 28 (1995), 2335-2352.

[65] R.L. Ricca, [a] Dynamics of knotted magnetic flux-tubes, in: XIX Int. Cong. Theor. Appl. Mech. - Abstracts, IUTAM, Kyoto, 1996, 94.

[66] R.L. Ricca, [b] The contributions of Da Rios and Levi-Civita to asymptotic potential theory and vortex filament dynamics, Fluid Dyn. Res. 18 (1996), 245-268.

[67] R.L. Ricca and M.A. Berger, Topological ideas and fluid mechanics, Phys. Today 49, 12 (1996), 24-30.

[68] R.L. Ricca and H.K. Moffatt, The helicity of a knotted vortex filament, in: Topological Aspects of the Dynamics of Fluids and Plasmas, H.K. Moffatt et al. (ed.), Kluwer, Dordrecht, The Netherlands, 1992, 225-236.

69] D. Rolfsen, Knots and Links, Publish or Perish Inc., Berkeley (CA), 1976.

[70] A. Schilham, Work done at the Department of Mathematics, University College London, 1995.

[71] T. Schlick and W. Olson, Trefoil knotting revealed by molecular dynamics simulations of supercoiled DNA, Science 257 (1992), 1110-1115.

[72] J.K. Simon, Energy functions for polygonal knots, in: Random Knotting and Linking, K.C. Millett \& D.W. Sumners (ed.), World Scientific, Singapore, 1994.

[73] De W. Sumners (ed.), New Scientific Applications of Geometry and Topology, PSAM 45, Am. Math. Soc., Providence, 1992.

[74] P.G. Tait, On Knots I, II, III, Scientific Papers 1, Cambridge University Press, 1898, 273-347.

[75] F. Tanaka and H. Takahashi, Elastic theory of supercoiled DNA, J. Chem. Phys. 83 (1985), 6017-6026.

[76] P. Traczyk, A new proof of Markov's braid theorem, this volume.

[77] A.V. Tur and V.V. Yanovsky, Invariants in dissipationless hydrodynamic media, J. Fluid Mech. (1993), 67-106.

[78] T. Uezu, Topological structure in flow systems, Prog. Theor. Phys. 83 (1990), 850-874.

[79] M. Van Dyke, An Album of Fluid Motion, The Parabolic Press, Stanford (CA), 1988.

[80] H. Villat, Lectures on the Theory of Vortices, Gauthier-Villars, Paris, 1930 (in French). 
[81] P. Vogel, Representation of links by braids: a new algorithm, Comment. Math. Helvetici 65 (1990), 104-113.

[82] M. Wadati and H. Tsuru, Elastic model of looped DNA, Physica D 21 (1986), 213-226.

[83] J.H. White, Self-linking and the Gauss integral in higher dimensions, Am. J. Math. 91 (1969), 693-728.

[84] J.H.C. Whitehead, An expression of Hopf's invariant as an integral, Proc. Nat. Acad. Sci. U.S.A. 33 (1947), 117-123.

[85] L. Woltjer, A theorem on force-free magnetic fields, Proc. Natl. Acad. Sci. USA 44 (1958), 489-491. 